\title{
Erratum: Study on dynamic response of track structures under a variable speed moving harmonic load
}

\author{
Yan Zhang', Yan Qi Liu ${ }^{2}$, Chun Fang Song ${ }^{3}$, Long Long $\mathrm{Xu}^{4}$ \\ ${ }_{1,3,4}$ School of Mechanical Engineering, Jiangnan University, Wuxi, China \\ ${ }^{2}$ Key Laboratory of Environment Noise and Vibration, Beijing Municipal Institute of Labor Protection, \\ Beijing, China \\ ${ }^{2}$ Corresponding author \\ E-mail: ${ }^{1} 6150805016 @ v i p . j i a n g n a n . e d u . c n,{ }^{2} j d y q l i u @ 163 . c o m,{ }^{3}$ songcf@jiangnan.edu.cn, \\ 42463944738@qq.com
}

DOI https://doi.org/10.21595/vp.2017.19452

Check for updates

\section{Publisher's note regarding paper}

Zhang Yan, Liu Yan Qi, Song Chun Fang, Xu Long Long Study on dynamic response of track structures under a variable speed moving harmonic load. Vibroengineering PROCEDIA, Vol. 14, 2017, p. 214-219, https://doi.org/10.21595/vp.2017.19217.

\section{The description of the correction}

The author numbering was incorrect in the paper originally submitted and finally approved (after the acceptance) by the Authors.

Incorrect authors' list:

Yan Zhang ${ }^{1}$, Yan Qi Liu $^{2}$, Chun Fang Song ${ }^{3}$, Long Long $\mathrm{Xu}^{4}$

$1,3,{ }^{4}$ School of Mechanical Engineering, Jiangnan University, Wuxi, China

${ }^{2}$ Key Laboratory of Environment Noise and Vibration, Beijing Municipal Institute of Labor Protection, Beijing, China

${ }^{2}$ Corresponding author

E-mail: 16150805016@vip.jiangnan.edu.cn, 2jdyqliu@163.com, ${ }^{3}$ songcf@jiangnan.edu.cn, 42463944738@qq.com

Correct authors' list:

Yan Qi Liu ${ }^{1}$, Yan Zhang ${ }^{2}$, Chun Fang Song ${ }^{3}$, Long Long Xu ${ }^{4}$

${ }^{1}$ Key Laboratory of Environment Noise and Vibration,

Beijing Municipal Institute of Labour Protection, Beijing, China

${ }^{2,3,4}$ School of Mechanical Engineering, Jiangnan University, Wuxi, China

${ }^{1,3}$ Corresponding author

E-mail: 1jdyqliu@163.com, ${ }^{2} 6150805016 @$ vip.jiangnan.edu.cn, ${ }^{3}$ songcf@jiangnan.edu.cn, 42463944738@qq.com 